\title{
Bidding Strategically for Scheduling in Grid Systems
}

\author{
Babak-Naddaf* and Jafar-Habibi*
}

\begin{abstract}
Grid computing is a new technology which involves efforts to create a huge source of processing power by connecting computational resources throughout the world. The key issue of such environments is their resource allocation and the appropriate job scheduling strategy. Several approaches to scheduling in these environments have been proposed to date. Market driven scheduling as a decentralized solution for such complicated environments has introduced new challenges. In this paper the bidding problem with regard to resources in the reverse auction resource allocation model has been investigated and the new bidding strategies have been proposed and investigated.
\end{abstract}

Keywords: Grid Computing, Grid Scheduling, Resource Allocation, Auction Model

\section{Introduction}

Over the past few years the architecture of computational systems has evolved from local systems with lower capacity to more distributed, decentralized systems with higher computational power.

Grid systems - as the manifestation of such decentralized systems - have high applicability to computational sciences such as high energy physics, astronomy, bioinformatics, economic forecasting, and so forth. The main challenge posed by grid systems is their scheduling strategy, which should satisfy the system users and the relevant resource requirements.

Market-based scheduling, as one of the best distributed scheduling schemes for such distributed systems, has consisted of different market models such as auctions, commodity market, etc. Because of the distributed architecture, the auction model is an attractive subject for researchers in this area.

Different auction protocols for resource allocation and job scheduling such as FPSA (first price Sealed bid), and English, Dutch, and second price auctions have been investigated thus far. One of the newest auction models for economic scheduling in grid systems is the reverse-auction [6] model. In this model, resource providers (bidders) play the main role in trading between users and resource owners. This paper represents an attempt to investigate the impact of the different bidding strategy followed by the resource providers on the performance parameters of the grid systems. We have conducted extensive simulation on GridSim [12] to evaluate the performance of our bidding strategy. Part 2 presents the related work on grid resource allocation; Part 3 discusses the Bidding problem; Part 4

\footnotetext{
Manuscript received 10 March, 2009; accepted 29 April, 2009.

Corresponding Author: Babak-Naddaf

* Information Technology department sharif university of technology
} international campus (baabak_firend@yahoo.com, habibi@sharif.edu) presents the contributions; and Part 5 consists of an explanation of Mathematical modeling.

Thereafter, Part 6 consists of a study of The general grid architecture; Part 7 presents a performance evaluation of random bidding; Part 8 proposes (various) historical bidding strategies and an evaluation of their performance; Part 9 discusses The new bidding model and Part 10 investigates its properties; Part 11 compares our model with the direct auction model; and, finally, Part 12 presents the conclusion.

\section{Related Work}

Y. Liu in [15] describes grid scheduling as the following: "with a common Grid infrastructure, Grid scheduling is used to answer the following question: Given a set of Grid applications, how can they be scheduled over multiple decentralized resources?" In order to answer the question, two main approaches have been proposed so far: The first consists of centralized scheduling, whereby the centric entity will schedule jobs on geographically distributed systems; the second is market-driven grid scheduling, by which traditional economic models are applied to job scheduling in computational grids.

Most of the works in this area have investigated the performance of different market models in the grid scheduling context [1-3, 8, 10, 11, 16]. For example, the authors of [1] investigated the performance of different economic models for resource management in distributed systems. The combinatorial auction-based protocols for resource allocation were investigated in [10]. However we concentrate on the performance of the reverse auction model proposed by [6] from the bidders' point of view. M. D. Assuncao and R. Buyya in [6] investigated the communication demand of reverse auction protocols. 
The Authors of [5] also used a similar auction model for scheduling in grid systems. F. I. Popovici and J. Wilkes in [4] investigated the profit gained by the resource provider under certain scheduling strategies.

\section{Bidding Problem}

In the reverse auction resource allocation model the resources' (bidders') main goal is to increase their own profits. Here, we have assumed that resources are local bidders, which means that they are only participating in auctions that belong to our system (and not anywhere else). According to this assumption, the main goal of the resources is to increase their own profits without considering the amount of time during which the resource is busy. On the other hand, if the bidders are global (i.e. participating in other systems auctions), then they have to consider the amount of profit in relation to the amount of busy periods. Here, the question is : "how can we reach this defined goal (maximum possible profit)? The Profit of the resources depends on the arrival pattern of tasks (which determine the number of sequential auctions in a specific period of time), the number of jobs per task (which determine the number of simultaneous auctions in a specific period of time), the size of each job, the number of users, the number of resources; and, in particular, it depends on the bidding strategies of other resources. For the sake of simplicity, the bidder's options for bidding are limited to \{very high, high, medium, low, very low . This set classifies the values that can be bid by a specific bidder. This classification will make the comparison of biddings easier, and may be generalized as a more general form (a format with more classes of bidding values.

'Bidding strategy' is defined as the algorithm that a bidder follows to select a specific class of bidding for participation in an auction.

\section{Contributions}

We have proposed a bidding strategy by which the bidders who follow it will have the highest amount of profit compared to bidders who follow other bidding strategies (Other strategies are random, having different means and deviations, and historical predictors with different window slides). In addition, the simulation results show that when all of the bidders in the system follow our strategy the system will converge to the equilibrium point under which no bidder has an incentive to deviate (which here means that if one bidder changes its amount of bidding, it will decrease its own profit).

\section{Mathematical Model}

In this part, the analytical modeling of the system will be proposed:

Effective parameters:

Processing power: P.P (MIPS)

Job length: J.L (MI)

Expected number of jobs per task: Expect $(\mu)$

Auction duration: A.D

Arrival pattern of tasks: inter -arrival of $\mathrm{t}$

Bidding values: A, B, C, D, E

Budget-constraint: unlimited for the users

Time-constraint: resources during the processing time do not have permission to participate in the auctions

Number of auctions: $\mathrm{T}$

Low level scheduling: space shared

Number of processing elements per machine: 1

Number of machines per resource: 1

Auction protocols: FPSA (First Price Sealed bid Auction)

Number of rounds: 1

Number of bidders: $\mathrm{N}$

Number of bidders in each bidding class: $\mathrm{Nx}$

Assumption (1): jobs will be submitted to the system with the constant inter -arrival time.

We have defined $\underline{n}$ bidding class $(\mathrm{X})$ with the following logical relation. According to the FPSA protocol, a lower bidding value will win the auction.

$X_{1}<X_{2}<\ldots<X_{n}, \mathrm{n}=$ number of bidding class (1)

The value of a specific bidding class $m$ has been proposed by the following description: Value $\left(X_{m}\right)$.

The processing time (P.T) is defined as the period of time in which the processing element of a specific resource starts processing the first job in a queue of waiting resources until finishing the processing of the last job in the queue. The Processing time for bidders in a Y bidding class is computed as follows:

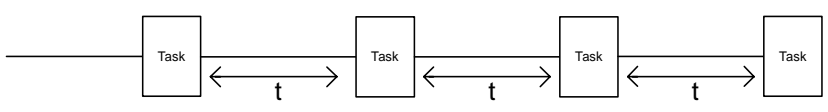

Fig. 1. Arrival pattern of tasks

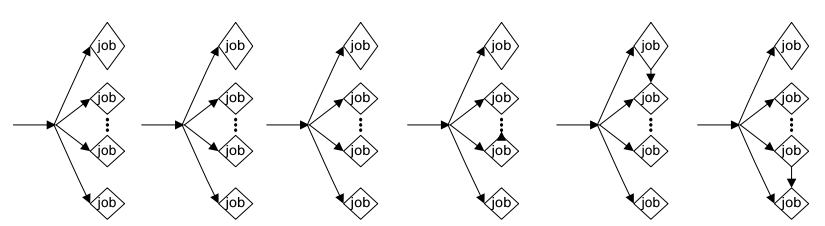

Fig. 2. Number of simultaneous and sequential auctions 


$$
{\mathrm{P} . \mathrm{T}_{y}}=\frac{1}{N_{y}} * \frac{J . L}{P . P} * \operatorname{Expect}(\mu)
$$

Ny: the number of bidders in a bidding class (Y).

Assumption (2): All the processing elements have the same P.P.

Assumption (3): None of the resources change their bidding values during $\mathrm{T}$ number of auctions. The following function eliminates the waiting time for a specific bidder until the next auction starts.

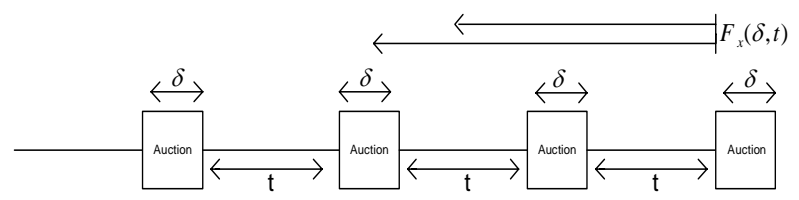

Fig. 3. Function F eliminates waiting time

$$
F_{x}(\delta, t)= \begin{cases}k \delta+k(t+1) & k \delta+k t<P T_{x}<k \delta+k(t+1) \\ k t+(k-1) \delta & k t+(k-1) \delta<P T_{x}<k t+k \delta\end{cases}
$$

We define profit $t_{x}$ as the profit of the resources in bidding class $\mathrm{X}$, wherein the profit of the resource is the number of winning auction multiplied by the bidding value. Therefore, the lowest bidding value will win all of the auctions for which it has the opportunity to bid. So, profit $_{1}$ has the following formula:

$$
\text { profit }_{1}=\frac{t(T-1)+T * \delta}{F_{1}(\delta, t)+\delta} * \text { value }\left(X_{1}\right)
$$

The profit of the resources in the other bidding classes is the number of participating auctions in which the bidders with lower bidding values do not have permission to bid multiplied by the bidding value of that class of bidding. To make the number of winning auctions predictable, we assume:

$$
\text { Assumption (4): P.T } \mathrm{T}_{y}<\mathrm{P} . \mathrm{T}_{y-1} \quad \forall y \in\{2 \ldots n\}
$$

According to assumption (2) and assumption (4) and formula (2), the number of bidders in each bidding class is greater than the number of bidders in the immediate next lower bidding value, so the profit of the bidders in bidding class 2 will be computed as follows:

$$
\text { profit }_{2}=\left(\frac{F_{1}(\delta, t)-(t+\delta)}{F_{2}(\delta, t)+\delta}\right) *\left(\frac{t(T-1)+T * \delta}{F_{1}(\delta, t)+\delta}\right) * \text { value }\left(X_{2}\right)
$$

By With extending this formula for profit $_{n}$, we can say:

$$
\operatorname{profit}_{n}=\left(\frac{F_{n-1}(\delta, t)-(t+\delta)}{F_{2}(\delta, t)+\delta}\right) \frac{\text { profit }_{n-1}}{\operatorname{value}\left(X_{n-1}\right)} \quad \mathrm{n} \neq 1
$$

Formula (4) will compute the profit of the resource in the nth bidding class, under the model assumptions. To maximize the profit of the resources we can use derivation in different dimensions; for example, if we are interested in finding the optimum job length for the profit of the resources, we have to optimize the profit of $n$ resources simultaneously. In the future, we would like to work on the mathematical solutions of this formula.

\section{Simulation Model}

The Simulation model follows the reverse auction model proposed in [6] for scheduling in the basic grid model. In the basic model (Fig. 4.) the users submit jobs according to the Poisson distribution. The Number of users that submit the tasks to the brokers in our simulation configuration is 20 , and the number of resources is 6. Every user has a dedicated broker who serves as an auctioneer with the responsibility of finding the best fit most suitable resource provider for the user. Here in the basic model it is assumed that different resources have the same properties, so the resource provider which presents a smaller than the others to execute the job will win the auction.

In the basic grid model, the job scheduling of the resources will be space -shared (the accepted jobs will be executed by the resource based on the FIFO queue structure). The duration of all the auctions is assumed to be the same, and the auction protocol is one round first price sealed bid auction. Another considerable point with regard to simulation setting is the limitation on bidding for resource providers, on the assumption that the resource providers that are processing the submitted jobs do not have permission to participate in any auctions. This

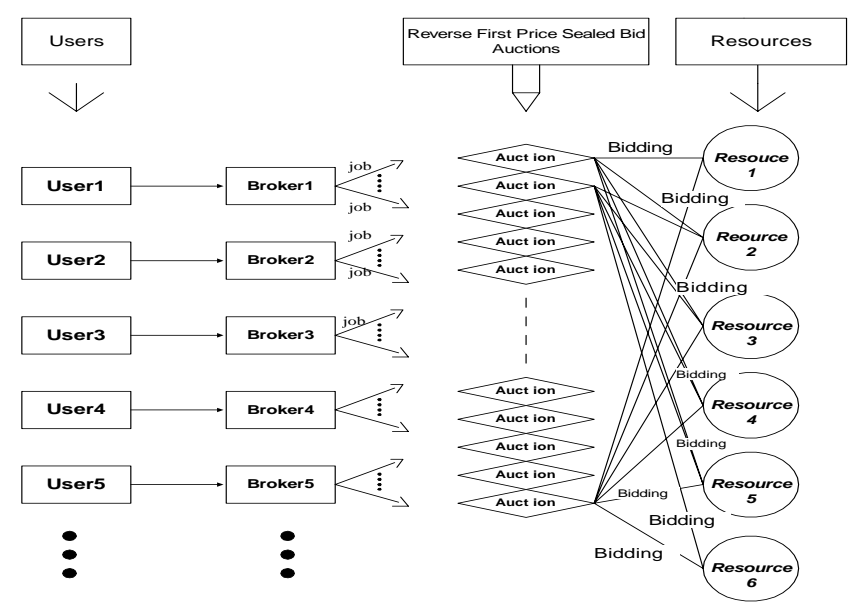

Fig. 4. General grid auction model 
limitation eliminates the resources with the long queues of accepted job which are waiting to be executed. On the other hand, the limitation on bidding while processing is an acceptable estimation of the deadline constraints of the jobs.

\subsection{Implementation of Bidding Strategies}

In the basic model we have classified the amount of money that can be offered by each bidder.There exist five classes of bidding, namely $\mathrm{A}, \mathrm{B}, \mathrm{C}$, and $\mathrm{E}$. each class represents the group of possible amounts that can be bid.

For example, if the highest amount of the bidding is equal to 100 and the lowest to 0 , then $\mathrm{A}$ will be equal to 90, $\mathrm{B}$ will be equal to 70 , C will be equal to 50,D will be equal to 30 , and $E$ will be equal to 10 . So the bidding range will be divided into 5 parts, where every class represents the average number of elements which belong to this part.

\subsection{Random Bidding Strategies}

The simplest strategy for bidding in an auction system is the random strategy. With this strategy, historical data on the bidding behaviors of the other resources and the rate of incoming jobs (arrival pattern under which the users submit tasks) will not be taken to account.

Based on the selection probability of bidding classes four random bidding strategies will be defined. The First one is shown in Fig. 5 (Random strategy_1). This random strategy selects the possible bidding class based on the uniform probability distribution. With this strategy, the probability of selecting $\mathrm{A}, \mathrm{B}, \mathrm{C}, \mathrm{D}$, or $\mathrm{E}$ for incoming auctions is the same.

In the second sub -class shown in Fig. 6, the highest value for bidding (A) has the highest probability of being selected for bidd3ing in an incoming auction (random strategy_2). This probability will decrease when the candidate bidding value decreases, so that $\mathrm{E}$ has the lowest

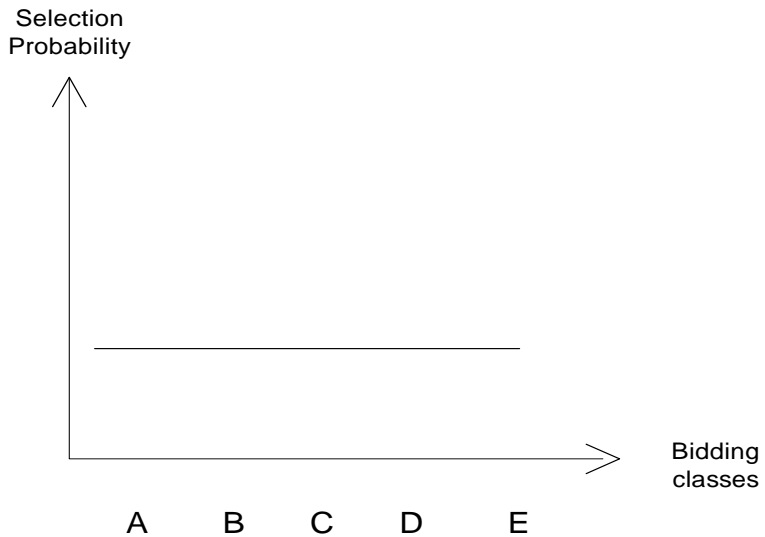

Fig. 5. Random strategy_1 probability of being selected.

Fig. 7. shows the third random strategy (random strategy_3), which selects the mean of the bidding values (class C) with the highest probability, and so the probability of selecting higher or lower bidding values will decrease.

Finally, in the fourth sub -class (random strategy_4), the lowest bidding value (E) has the highest probability of being chosen, and, by increasing the value of the bidding alternatives (D, C, B, A), the possibility of selection will decrease (Fig. 8.).

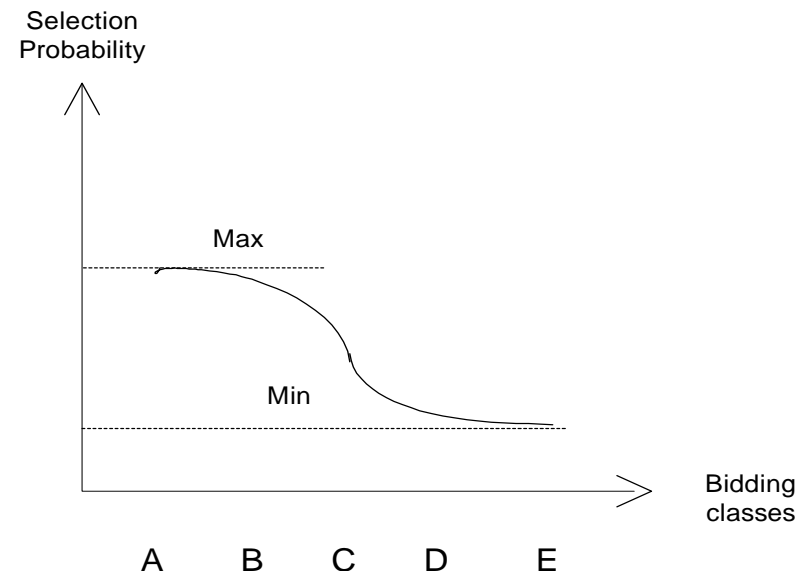

Fig. 6. Random strategy_2

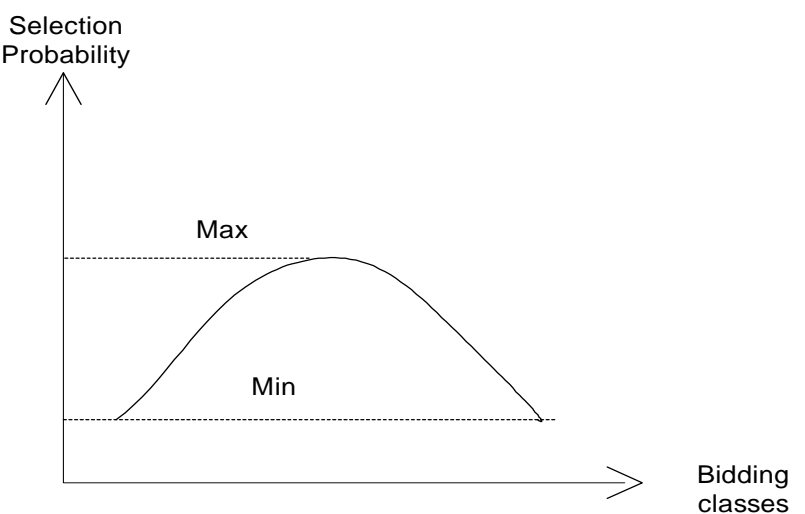

A $\quad$ B $\quad$ C $\quad$ D $\quad E$

Fig. 7. Random strategy_3

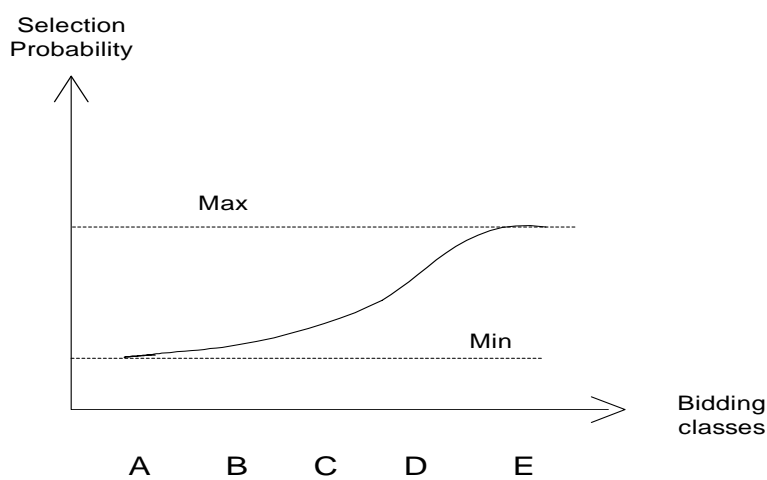

Fig. 8. Random strategy_4 


\section{Performance Evaluation of the Random Bidding Strategies}

The results of simulation with 4 random bidding resources are shown in following. The impact of the job sizes and the number of jobs in each task has been investigated. Note in Fig. 9 (and in Figs 10, 11, 12, and 13) that resources bid based on the following strategies: Resource 1 bids with a high value (random strategy_2); Resource 2 follows bidding with a low value (random strategy_4); Resource 3 prefers bidding with a medium value (random strategy_3); and Resource 4 uses bidding randomly with uniform probability (random strategy_1). The 3D bar chart in Fig. 9 shows that when the number of jobs per task is low (and where, consequently, the number of simultaneous auctions will be low), the resource which is interested in bidding lower will gain more profit, whereas when the number of simultaneous auctions increases, the profits of the resource which bids higher will increase (Figs 10 and 11). And, finally, in the next charts (Fig. 12 and Fig. 13), when uncertainty is added to the number of jobs per task, the deviation in the amount of gained profit will increase. These charts show very clearly that when the number of arrival auctions and job sizes

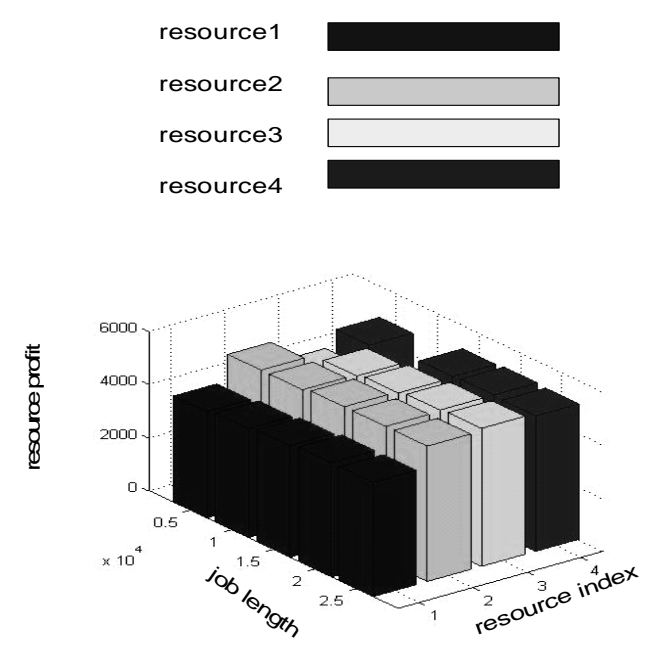

Fig. 9. Number of jobs per task=1

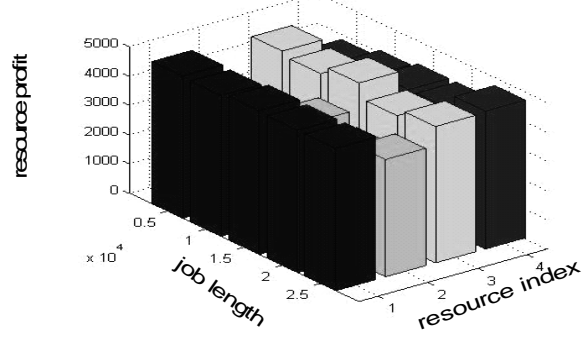

Fig. 10. Number of jobs per task=5

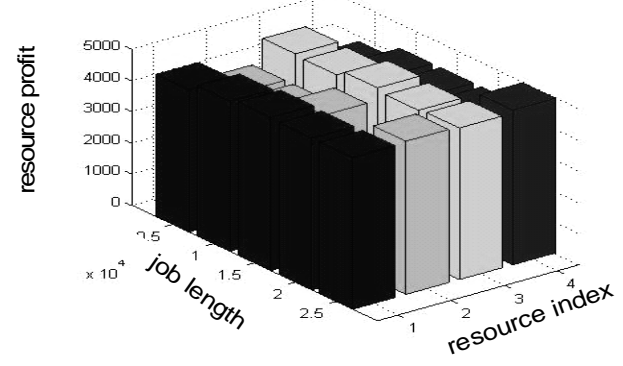

Fig. 11. Number of jobs per task=10

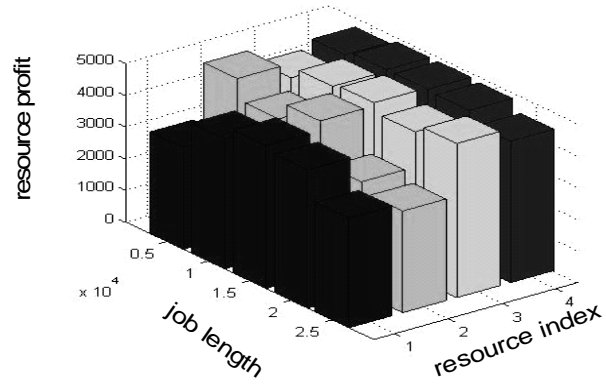

Fig. 12. Number of jobs per task=random value between 1 and 5

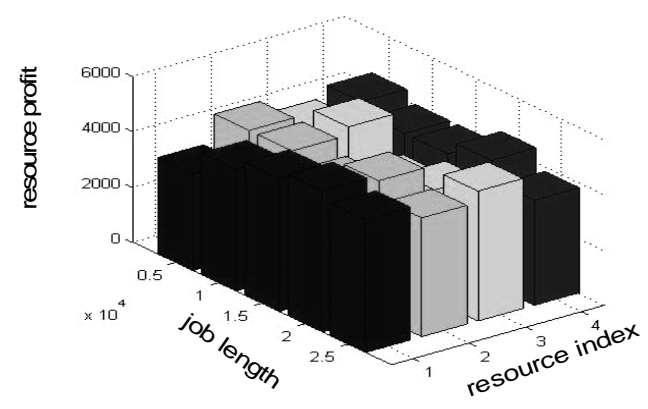

Fig. 13. Number of jobs per task is a random value from 1 to 10

increase, the profits gained by the four random strategies will converge to the same value.

\section{Historical Strategies}

In this part we will investigate three main bidding strategies that offer the next bidding value based on the previous winner's values. The First one was inspired by a known linear predictor ES (exponential smoothing). Our bidding strategy suggests the winner's value at the last auction as the bidding value for the next incoming auction. The performance of this strategy is shown in Fig. 14. (One resource follows this historical strategy, while the other resources follow one of the 4 random bidding strategies). In Fig. 14, the length of each job is 5000 , while resource 1 follows random strategy_2; resource2 selects bidding 
values based on random strategy_3; resource3 follows random strategy_1; resource4 bids based on random strategy_4; and, finally, resource5 follows our first historical strategy (ES inspired strategy) for participation in auctions. it is clear in Fig. 14 that when the number of auctions increases the profit of resource5, which follows the historical strategy, will increase more than that of the other resources. The next bidding strategy investigated uses the idea of Auto Regression to predict the next winning value.

This predictor conducts forecasts using the windows of previous data (always starting with the most recent data and working backwards in time) [14]. Based on this strategy, the bidder chooses the value that has the highest number of wins in the previous auctions as the best bidding value for the incoming auction. The performance of this bidding strategy is compared with the previous one in Fig. 15. Fig. 15 shows the change in the bidding strategy of resource 2 to the second historical bidding strategy. Other configurations are the same as those shown in Fig. 14.

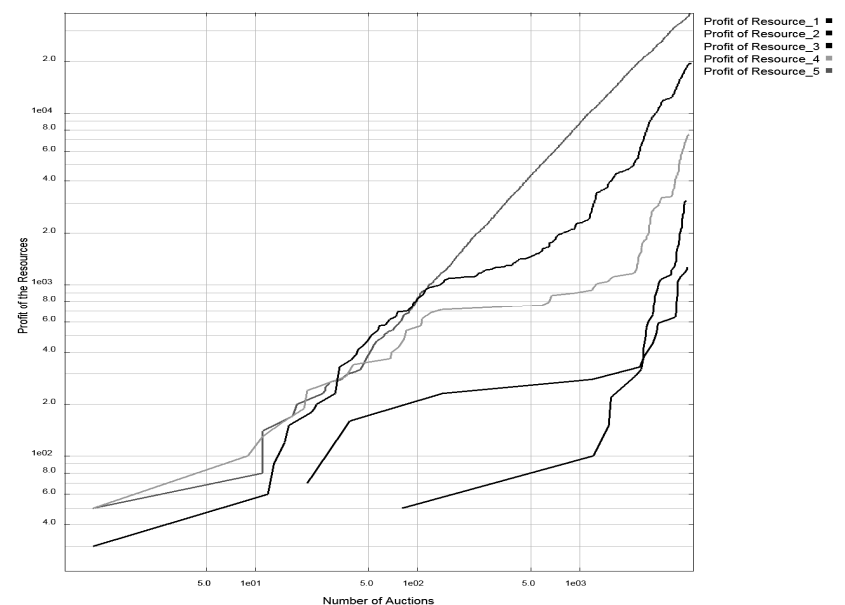

Fig. 14. Performance of the first historical bidding strategy

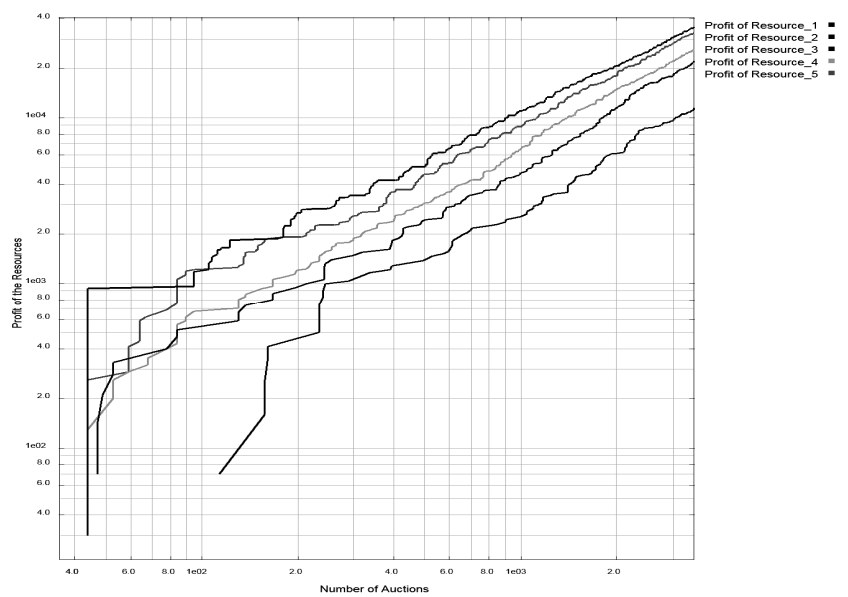

Fig. 15. Performance of the second historical bidding strategy compared with the first bidding strategy
The main problem with the second historical bidding strategy is that it decides based on the number of times that one bidding class has won, whereas it is possible for a specific higher class with a lower number of wins to have a higher amount of gained profit. Based on the size of the window the performance of this strategy will change. In systems with low variability and uncertainty, these two predictors work just like each other, but when uncertainty increases the performance of these two predictors will change. To impose more variability on our simulation, we have assumed that resources (bidders) participate in the auctions randomly. (The bidders waiting time after receiving CFP, is distributed uniformly.)

\section{Proposed Game Model}

This game model has defined a common token for bidders so that each bidder interested in changing his bidding class (A, B, C, D, E) should own the token. The token will be handed to the bidders (resource providers) sequentially after a specific number of auctions $(\mathrm{N})$. The sequential auction model is shown in Fig. 16. Based on this architecture, we propose a bidding algorithm (greedy bidding algorithm) which is expressed in the following part.

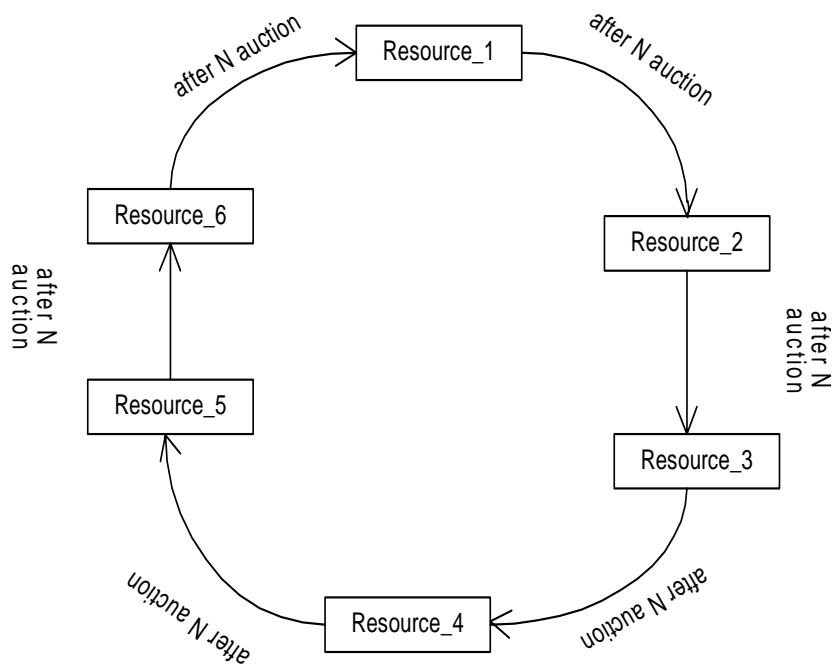

Fig. 16. Token will be delivered to the resources after $\mathrm{N}$ action

Greedy bidding algorithm:

1. Wait until gaining the token, and then compare the profits of the resources in the previous $\mathrm{N}$ number of auctions.

2. Change your bidding class to the bidding class of the resource that has the highest amount of profit. If the set of the resources with the gained profit exists, consider 
the highest value of the profit as the profit of this class of bidding.

If all resources follow the same bidding class, it may be a trap, whereupon you have to change to the random bidding class; this change can be done intelligently

\section{Comparisons between Bidding with the Game Model and without the Game Model}

Simulations show that there exist sets of bidding classes that obtain the best profit of all the resources. On the other hand, when different parameters of the system (such as the tasks arrival rate) are fixed, then there exist such as (X, Y, $\mathrm{Z}, \mathrm{K}, \mathrm{M}$, and $\mathrm{N}$ ) for six resources where no resource has the better choice. Variables $\mathrm{X}, \mathrm{Y}, \mathrm{Z}, \mathrm{K}, \mathrm{M}$, and $\mathrm{N}$ represent the bidding classes that can be substituted. In other words, when all bidders follow our greedy bidding strategy the profit of the resources will reach the point where if one resource tries to use another bidding class (other than the one suggested by the greedy strategy), then that resource will lose profit while the profit of the other resources will increase. The convergence property of this game model is shown in Fig. 17.
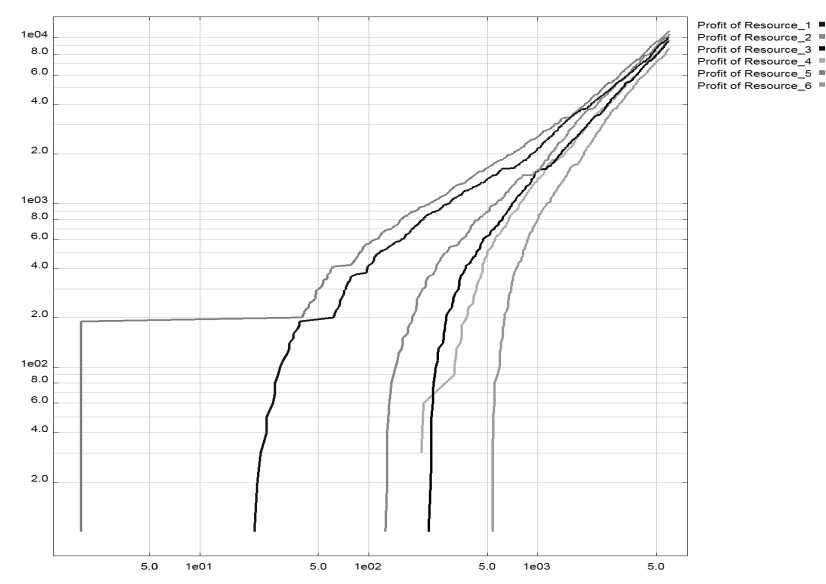

Fig. 17. Convergence property of the greedy bidding strategy

We have compared the profit gained by resources when they follow our game model and when they do not follow our game model for bidding in the reverse auction grid systems. The experiments were repeated five times; with the maximum, minimum, and average amount of gained profit proposed in table 1 and table 2 . In the simulation with our game model, when 6 resources use the greedy bidding strategy, the size of the jobs varies between [5000...10000], the number of jobs per task is one; and the profit of the six resources when the number of auctions reaches 2000 is as follows:
Table 1. Profit of the resources when following the greedy bidding strategy

\begin{tabular}{|c|c|c|c|c|}
\hline Res. No & $\begin{array}{c}\text { Average } \\
\text { profit }\end{array}$ & $\begin{array}{c}\text { Maximum } \\
\text { deviation }\end{array}$ & $\begin{array}{c}\text { Bidding } \\
\text { strategy }\end{array}$ & $\begin{array}{c}\text { Initial } \\
\text { bidding } \\
\text { class }\end{array}$ \\
\hline \hline Resource1 & 12200 & $\begin{array}{l}12700 \\
11400\end{array}$ & $\begin{array}{c}\text { Greedy } \\
\text { bidding } \\
\text { strategy }\end{array}$ & C \\
\hline Resource2 & 21000 & $\begin{array}{l}21400 \\
20500\end{array}$ & $\begin{array}{c}\text { Greedy } \\
\text { bidding } \\
\text { strategy }\end{array}$ & C \\
\hline Resource3 & 16000. & 17000 & $\begin{array}{c}\text { Greedy } \\
\text { bidding } \\
\text { strategy }\end{array}$ & C \\
\hline Resource4 & 14400. & 14500 & $\begin{array}{c}\text { Greedy } \\
\text { bidding } \\
\text { strategy }\end{array}$ & C \\
\hline Resource5 & 14500. & 14720 & $\begin{array}{c}\text { Greedy } \\
\text { bidding } \\
\text { strategy }\end{array}$ & C \\
\hline Value & 15130 & 15600 & $\begin{array}{c}\text { Greedy } \\
\text { bidding } \\
\text { strategy }\end{array}$ & C \\
\hline Resource6 & 12700. & 133000 & 12000 & II. \\
\hline & 1400 & & 14500 & \\
\hline
\end{tabular}

Table 2. Profit of the resources when not following the greedy bidding strategy

\begin{tabular}{|c|c|c|c|c|}
\hline Res. No & $\begin{array}{c}\text { Average } \\
\text { profit }\end{array}$ & $\begin{array}{c}\text { Maximum } \\
\text { deviation }\end{array}$ & $\begin{array}{c}\text { Bidding } \\
\text { strategy }\end{array}$ & $\begin{array}{c}\text { Initial } \\
\text { bidding } \\
\text { class }\end{array}$ \\
\hline \hline Resource1 & 14800 & $\begin{array}{c}15000 \\
14500\end{array}$ & $\begin{array}{c}\text { First } \\
\text { historical } \\
\text { strategy }\end{array}$ & C \\
\hline Resource2 & 7800 & 8400 & $\begin{array}{c}\text { Random } \\
\text { strategy4 }\end{array}$ & C \\
\hline Resource3 & 3200. & $\begin{array}{l}3800 \\
2600\end{array}$ & $\begin{array}{c}\text { Random } \\
\text { strategy1 }\end{array}$ & C \\
\hline Resource4 & $850 .$. & $\begin{array}{c}1300 \\
700\end{array}$ & $\begin{array}{c}\text { Random } \\
\text { strategy3 }\end{array}$ & C \\
\hline Resource5 & 14100. & $\begin{array}{l}14300 \\
13600\end{array}$ & $\begin{array}{c}\text { Random } \\
\text { strategy2 }\end{array}$ & C \\
\hline Resource6 & 18000. & $\begin{array}{l}18200 \\
17400\end{array}$ & $\begin{array}{c}\text { Second } \\
\text { historical } \\
\text { strategy }\end{array}$ & C \\
\hline Mean value & 9700 & $\begin{array}{l}10200 \\
9300\end{array}$ & \\
\hline
\end{tabular}




\subsection{Other Performance Parameters}

Two of the important performance parameters for a grid system are the job success rate (number of jobs that finishes their processing before their lost their deadline to the total number of submitted jobs) and the average turnaround time per job (which means the average time from job submission time until the results begin to return to the user). The comparison between the job success rates of bidders who follow different bidding strategies is given in Fig. 18.

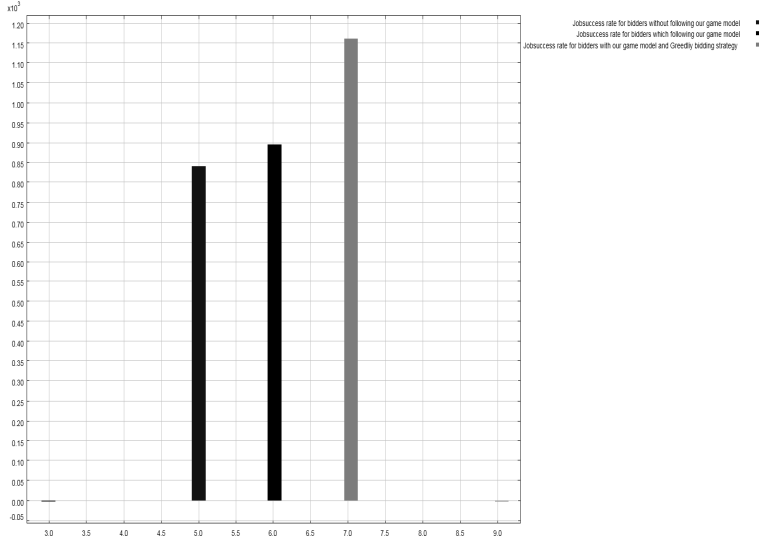

Fig. 18. Comparison of job success rate The average turnaround time of jobs for resources with different biddings is proposed in Fig. 19.

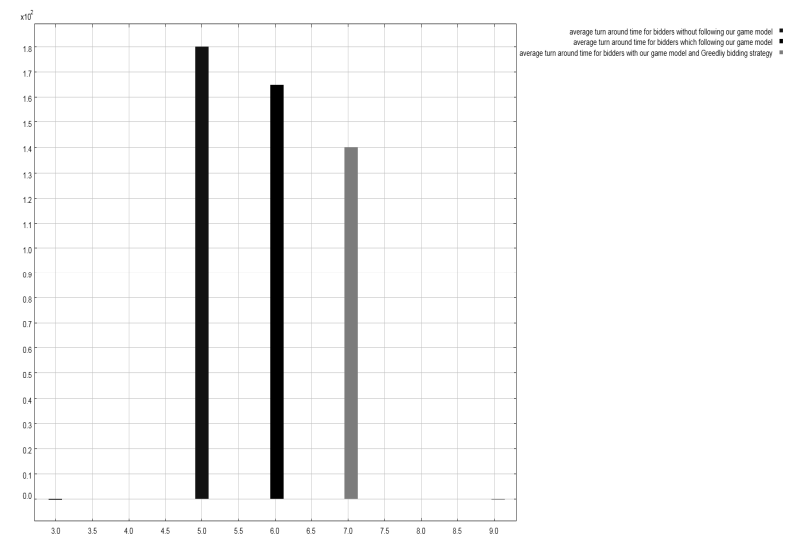

Fig. 19. The average turnaround time of jobs for resources following different bidding strategies.

\section{Comparison with the Direct Auction Model}

Finally, we compared our reverse auction model with the direct auction model. Direct auction resource allocation has been proposed completely in [13]. We have applied configuration of [13] (such as the number of resources, users, and their properties) for our simulation. job length in this experiment varies from $10000 \mathrm{MI}$ to $20000 \mathrm{MI}$. In the direct model, users participate in the auction as bidders. There are three famous resource selection policies for users: TimeOptimized, BudgetOptimized, and Random. With the TimeOptimized policy, the user tries to minimize the average turnaround time of a job; with BudgetOptimized, the user will try to minimize the average budget spent per job; and in Random the user selects resources randomly. The comparison of these three resource selection policies with the reverse auction model is shown in Fig. 19 and Fig. 20. To justify this effect we can say that when the resources follow the greedy bidding strategy, the average number of jobs in the waiting queues of the resources show the least deviation. So, when the incoming jobs have different deadline constraints, the possibility of losing the deadline will decrease. (On the other hand, the average time a job spends in the waiting queue is least when all the resources are bidding greedy).

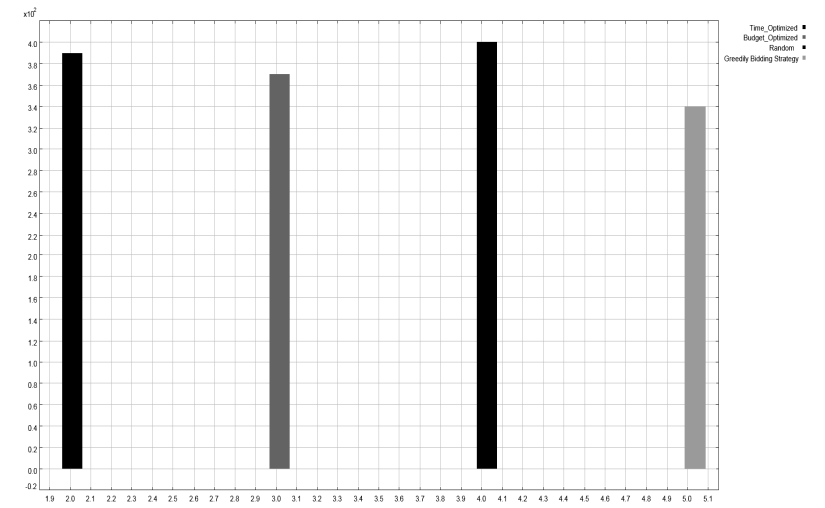

Fig. 19. Job success rate in the auction and reverse auction models.

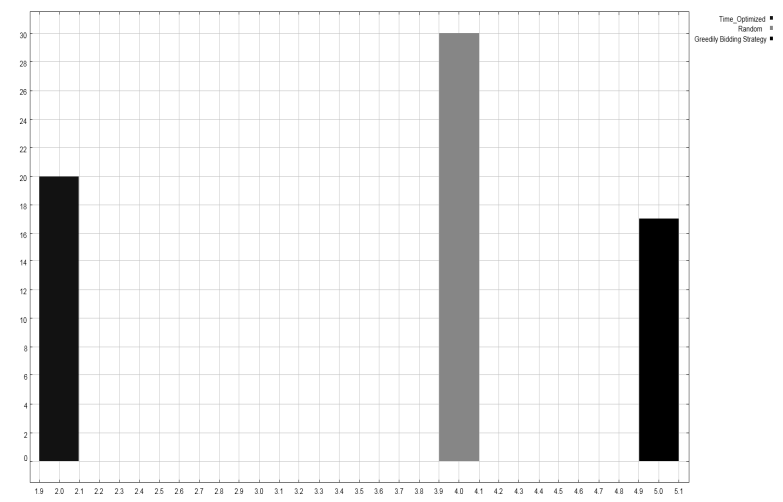

Fig. 20. Average turnaround time per job in the auction and reverse auction models.

\section{Conclusions and Future Work}

For this project different bidding strategies were evaluated and compared from the resource providers' point 
of view. The Main performance parameter is the profit gained by different resources. A game model and greedy bidding strategy were proposed, we have shown when all the resources follows greedy bidding strategy the highest amount of the profit will be gained by the resources. Finally, we compared our grid model with the grid direct auction model. In the future, we hope to expand the basic game model to resources and users with different capabilities, and we would also like to find an analytical solution for the calculated recursive equation discussed in part 5.

\section{References}

[1] R. Buyya, D. Abramson, J. Giddy, and H. Stockinger, "Economic Models for Resource Management and Scheduling in Grid Computing," J. Concurrency and Computation: Practice and Experience (CCPE), Wiley Press, USA, May, 2002.

[2] R. Buyya, D. Abramson, and J. Giddy, "Economy driven resource management architecture for computational power grids," In PDPTA '00: International Conf. on Parallel and Distributed Processing Techniques and Applications, 2000.

[3] R. Buyya, D. Abramson, and S. Venugopal, "The grid economy," Proc. of the IEEE, Vol.93, No.3, pp.698714, March, 2005.

[4] F. I. Popovici, J. Wilkes, "Profitable services in an uncertain world," In SC '05: Proc. of the 2005 ACM/ IEEE conf. on Supercomputing, IEEE Computer Society, pp.36, Washington, DC, USA, 2005.

[5] M. Dalheimer, F. Pfreund, and P. Merz, "Agent-based grid scheduling with calana," In Proc of the Second Grid Resource Management Workshop (GRMW 2005), Poznan, Poland, 2005.

[6] M. D. Assuncao, R. Buyya, "An Evaluation of Communication Demand of Auction Protocols in Grid Environments," Proc of the 3rd International Workshop on Grid Economics \& Business (GECON 2006), World Scientific Press, May 16, 2006, Singapore.

[7] R.P. McAfee and J. McMillan, "Auctions and bidding," J. Economic Literature, Vol.25, No.2, pp.699-738, 1987.

[8] D. Grosu, A. Das, "Auction-Based Resource Allocation Protocols in Grids," International Conference on Parallel and Distributed Computing and Systems, Nov., 2004, pp.20-27.
[9] R. Buyya, D. Abramson, and J. Giddy, "Nimrod/G: An Architecture for a Resource Management and Scheduling System in a Global Computational Grid,” International Conference on High Performance Computing in Asia-Pacific Region, May, 2000.

[10] A. Das, D. Grosu, "Combinatorial Auction-Based Protocols for Resource Allocation in Grids," IEEE International Parallel and Distributed Processing Symposium (IPDPS'05), 2005.

[11] U. Kant, D. Grosu, "Double Auction Protocols for Resource Allocation in Grids," International Conference on Information Technology: Coding and Computing (ITCC'05), 2005, Vol.1, pp.366-37.

[12] R. Buyya, M. Murshed, "GridSim: A Toolkit for the Modeling and Simulation of Distributed Resource Management and Scheduling for Grid Computing," $J$. Concurrency and Computation: Practice and Experience (RCCPE), Vol.14, Issue13-15, Wiley Press, Nov.-Dec., 2002.

[13] S. R. Reddy, "Market Economy Based Resource Allocation in Grids," Master Thesis, Indian Institute of Technology, Kharagpur, India, May, 2006.

[14] M. dobber, R.V. Mei, G. koole, "Developing New Prediction Method for Job Running times on Shared Processors: Survey, Statistical Analysis and New Avenues”, J. performance evaluation, pp.755-781, No.64, 2007.

[15] Y. Liu, "Grid Scheduling," Department of Computer Science University of Iowa. yanliu@cs.uiowa.edu

[16] G. Stuer, K. Vanmechelen, J. Broekhove, "A commodity market algorithm for pricing substitutable grid resource”, J. future generation computer systems, pp.688-701, No.23, 2007. 


\section{Jafar Habibi}

Associate Professor, Ph.D. in Computer Science, 1998, University of Manchester, UK. Currently he is the faculty member of sharif university of technology. His research interest is simulation, software engineering, information systems, system analysis and design

\section{Babak-Naddaf}

He received the BS degrees in computer engineering Azad University of Najaf Abad and he is undertaking MS degree in Sharif University of technology. His interesting subject is performance evaluation of high performance computing and distributed systems. 